12.1;14

\title{
Применение электростатической силовой микроскопии для определения статической диэлектрической проницаемости индивидуальных молекул гемоглобина
}

\author{
() Н.А. Давлеткильдеев ${ }^{1,2}$, Д.В. Соколов ${ }^{1}$, Е.Ю. Мосур ${ }^{1,2}$, А.А. Лопандина ${ }^{2}$, В.В. Болотов ${ }^{1,2}$ \\ ${ }^{1}$ Омский научный центр СО РАН, Омск, Россия \\ ${ }^{2}$ Омский государственный университет им. Ф.М. Достоевского, Омск, Россия \\ E-mail: nadim@obisp.oscsbras.ru
}

Поступило в Редакцию 5 апреля 2019г.

В окончательной редакции 4 июня 2019г.

Принято к публикации 20 июня 2019 г.

Представлена методика определения диэлектрической проницаемости индивидуальных молекул гемоглобина на основе моделирования профилей их изображений, полученных методом электростатической силовой микроскопии. Полученные значения статической диэлектрической проницаемости согласуются с известными литературными данными. Предложенная методика может быть адаптирована для определения диэлектрических характеристик индивидуальных молекул различных белков.

Ключевые слова: гемоглобин, электростатическая силовая микроскопия, диэлектрическая проницаемость.

DOI: 10.21883/PJTF.2019.19.48313.17827

\begin{abstract}
Чувствительность электрических и диэлектрических свойств белков к изменению условий окружающей среды определяет перспективу их применения в качестве активного элемента биосенсора для измерения физических, химических, биологических или любых других параметров, влияющих на отклик биосенсора в виде изменения емкости, напряжения, сопротивления или тока [1]. Для более полного понимания влияния условий окружающей среды на указанные выше свойства белков необходима разработка экспериментальных методов определения их электрофизических параметров на уровне индивидуальных молекул для того, чтобы исключить эффекты коллективного взаимодействия в ансамбле молекул на процессы поляризации и перенос заряда в молекулах белка.
\end{abstract}

Выбор гемоглобина (Hb) в качестве объекта исследования в настоящей работе обусловлен тем, что он хорошо изучен и часто используется в качестве модельного глобулярного белка. Кроме того, Нb применяется в биосенсорике [2].

Уникальным методом исследования диэлектрических свойств индивидуальных микро- и нанообъектов является электростатическая силовая микроскопия (ЭСМ). Данный метод обладает высокой чувствительностью и латеральным разрешением и представляет собой мощный инструмент для исследования диэлектрического отклика и распределения электрического поля и зарядов на поверхности объектов субмикронных размеров. Возможность использования ЭСМ для количественного определения диэлектрического отклика одиночных бактериальных клеток с высокой точностью и воспроизводимостью продемонстрирована в [3]. Целью настоящей работы является развитие методики электростатической силовой микроскопии для определения диэлектрической проницаемости индивидуальных молекул гемоглобина.

Образец для исследования диэлектрического отклика индивидуальных молекул Нb готовился следующим образом. На расщепленную поверхность высокоориентированного пиролитического графита (ВОПГ) наносилось $25 \mu 1$ водного раствора $\mathrm{Hb}$ с концентрацией $70 \mu \mathrm{g} / \mathrm{ml}$, по истечении $20 \mathrm{~min}$ ВОПГ отмывался в деионизованной воде для удаления неадсорбированного белка и высушивался на воздухе.

ЭСМ-измерения образца проводились на атомносиловом микроскопе MFP-3D SA (Asylum Research, США) в Омском региональном центре коллективного пользования ОНЦ СО РАН. Для исключения влияния рельефа поверхности образца на результаты измерения использовалась двухпроходная методика: в каждой строке сканирования на первом проходе с помощью полуконтактного метода определялся рельеф поверхности, на втором зонд отводился от поверхности на заданное расстояние и осуществлялось повторное сканирование по траектории первого прохода. Регистрируемым сигналом на втором проходе являлся сдвиг фазы колебаний кантилевера, поверхностное распределение которого формирует ЭСМ-изображение.

Высота сканирования на втором проходе выбиралась из условия получения наилучшего отношения сигнал/шум на ЭСМ-изображении, которое достигается путем соблюдения баланса между высотой зонда над образцом и амплитудой колебаний кантилевера. При варьировании данных параметров опытным путем была определена оптимальная высота сканирования на втором проходе, которая составила $30 \mathrm{~nm}$.

На втором проходе к зонду прикладывалось постоянное напряжение смещения величиной $+3,+5,+7$, 
$a$

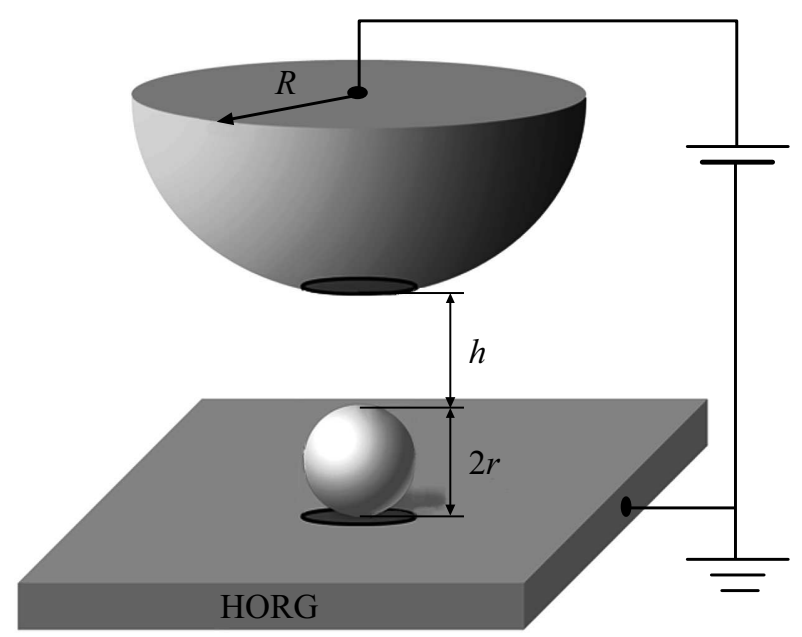

$b$

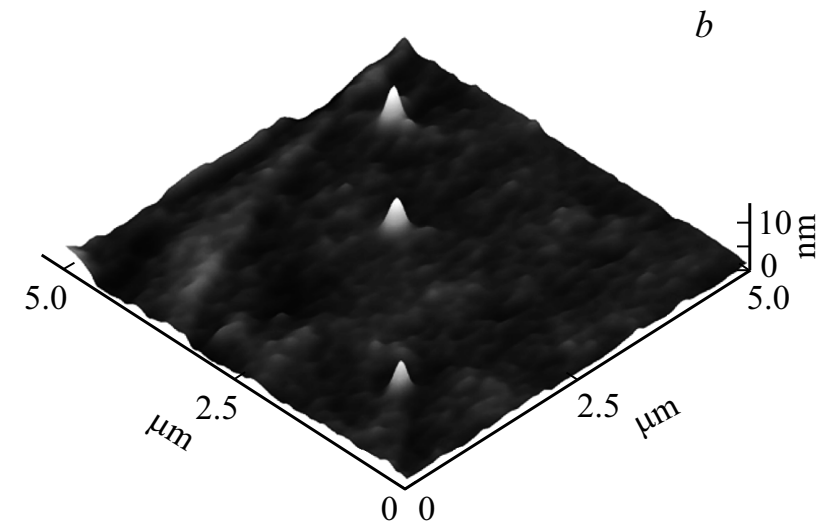

Pис. 1. Схематическое представление ЭСМ-эксперимента (a) и 3D-изображение поверхности ВОПГ с иммобилизованными индивидуальными молекулами $\mathrm{Hb}(b)$.

$-3,-5,-7 \mathrm{~V}$. В дальнейшем на основе зависимости величины ЭСМ-сигнала от приложенного напряжения определялась внешняя контактная разность потенциалов (КРП) между зондом и ВОПГ [4]. Учет КРП необходим для определения величины фактического напряжения между зондом и подложкой при расчете теоретического значения ЭСМ-сигнала и точного вычисления диэлектрической проницаемости $\mathrm{Hb}$.

Для исключения влияния адсорбата на величину диэлектрического отклика все измерения проводились в атмосфере сухого азота при относительной влажности $R H \sim 5 \%$. В работе использовались проводящие кантилеверы серии ETALON HA_FM (NT-MDT SI, Россия) c Pt-покрытием, имеющие следующие параметры: резонансная частота $\sim 100 \mathrm{kHz}$, радиус закругления зонда $\sim 35 \mathrm{~nm}$.

На рис. 1, $а$ представлена схема ЭСМ-эксперимента. На подложке ВОПГ расположена молекула гемоглобина, форма которой в рамках используемой модели принята за шар радиуса $r$. На постоянной высоте $h$ над образцом находится зонд с радиусом закругления острия $R$. Между зондом и подложкой прикладывается постоянное напряжение $U$.

Согласно [5,6], теоретическая разность фазовых сдвигов, характеризующая исследуемый объект, вычисляется по формуле

$$
\Delta \Phi_{\text {theor }}=\Phi-\Phi_{0}
$$

Подробно опишем величины, входящие в выражение (1): фазовый сдвиг колебаний кантилевера, обеспечиваемый емкостной связью зонд-подложка,

$$
\Phi_{0}=\operatorname{arctg}\left[\left(\frac{Q}{2 k}\right)(U-\Delta U)^{2} \frac{\partial^{2} C_{0}(z)}{\partial z^{2}}\right],
$$

a также сдвиг, определяемый емкостной связью зонд-молекула-подложка,

$$
\Phi=\operatorname{arctg}\left[\left(\frac{Q}{2 k}\right)(U-\Delta U)^{2} \frac{\partial^{2} C(z)}{\partial z^{2}}\right],
$$

где $\Delta U-$ КРП между зондом и подложкой, $Q=234-$ добротность кантилевера, $k=3.4 \mathrm{~N} / \mathrm{m}$ - жесткость кантилевера, $\frac{\partial^{2} C_{0}(z)}{\partial z^{2}}, \frac{\partial^{2} C(z)}{\partial z^{2}}-$ вторые производные

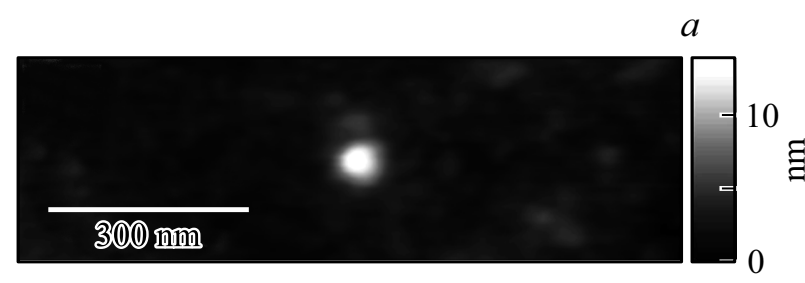
$\Xi$
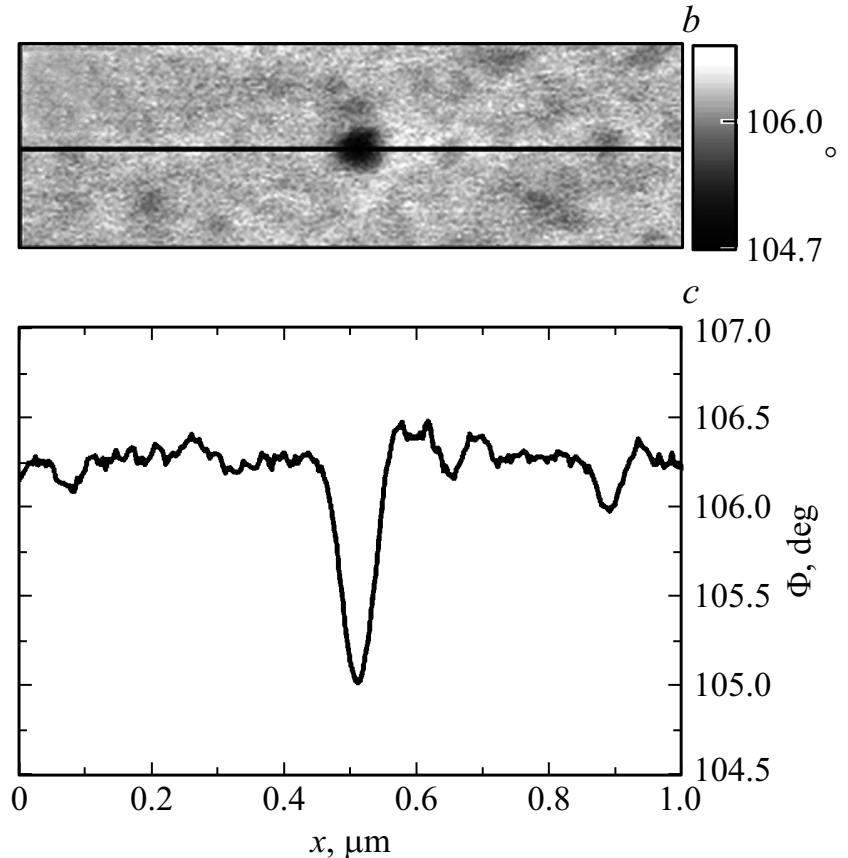

Рис. 2. $a-$ топографическое изображение молекулы $\mathrm{Hb}, b-$ соответствующее ЭСМ-изображение при напряжении на зонде $+7 \mathrm{~V}, c-$ профиль поперечного сечения ЭСМ-изображения. 


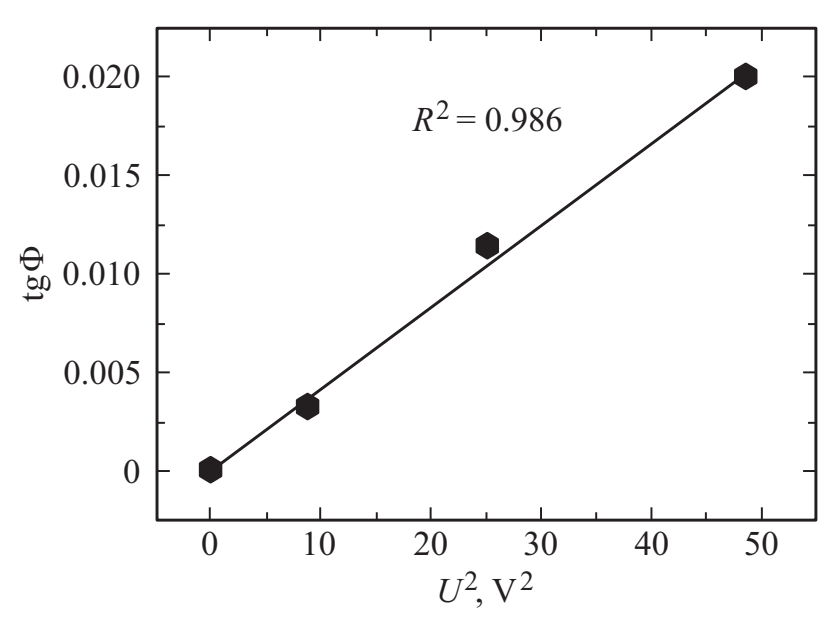

Рис. 3. Зависимость $\operatorname{tg} \Phi$ от $U^{2}$ для индивидуальной молекулы Нb диаметром $8 \mathrm{~nm}$.

соответствующих емкостей, вычисляемые по формулам

$$
\begin{aligned}
& \frac{\partial^{2} C_{0}(z)}{\partial z^{2}}=4 \varepsilon_{0} \int_{0}^{r} \int_{y_{1}}^{y_{2}}\left[h+R-\sqrt{R^{2}-x^{2}-y^{2}}\right]^{-3} d x d y \\
& \frac{\partial^{2} C(z)}{\partial z^{2}}=4 \varepsilon_{0} \int_{0}^{r} \int_{y_{1}}^{y_{2}}\left[h+R-\sqrt{R^{2}-x^{2}-y^{2}}\right. \\
& \left.+2\left(1-\frac{1}{\varepsilon}\right)\left(r-\sqrt{r^{2}-x^{2}-y^{2}}\right)+\frac{2 r}{\varepsilon}\right]^{-3} d x d y
\end{aligned}
$$

Здесь $\varepsilon_{0}$ - электрическая постоянная, $\varepsilon-$ диэлектрическая проницаемость молекулы гемоглобина, $y_{1}, y_{2}-$ пределы интегрирования по оси ординат, равные $-\sqrt{r^{2}-x^{2}}$ и $\sqrt{r^{2}-x^{2}}$ соответственно.

С помощью выражений (1)-(5) определялась диэлектрическая проницаемость молекул Нb путем подгонки теоретической разности фазовых сдвигов к аналогичной величине, измеренной экспериментально.

На рис. 1, $b$ представлено 3D-изображение поверхности ВОПГ с иммобилизованными индивидуальными молекулами Нb. По топографическим изображениям (рис. 2,a) определялась высота молекул $\mathrm{Hb}$, а на ЭСМ-изображениях (рис. 2,b) проводился профиль поперечного сечения для вычисления разницы в сдвиге фаз между молекулой и подложкой (рис. 2,c).

Для проверки того, что контраст ЭСМ-изображений молекулы $\mathrm{Hb}$ обусловлен емкостной связью зонд-образец, а не связан с наличием свободных зарядов на молекуле белка, была построена зависимость $\operatorname{tg} \Phi$ от $U^{2}$ (рис. 3). Строгая линейная зависимость свидетельствует о том, что контраст ЭСМ-изображений молекул гемоглобина определяется исключительно емкостной связью зонд-образец.

На основе моделирования профилей ЭСМ-изображений индивидуальных молекул Нb определено среднее значение диэлектрической проницаемости белка, которое с учетом разброса равно $2.9 \pm 0.9$. По литературным данным диэлектрическая проницаемость белков лежит в диапазоне 2-4 [7-9], что свидетельствует об адекватности полученных значений.

Таким образом, в работе продемонстрирована методика определения статической диэлектрической проницаемости индивидуальных молекул гемоглобина на основе количественного анализа профилей их ЭСМ-изображений с использованием модели электростатического взаимодействия между зондом атомносилового микроскопа и молекулой белка. При использовании указанной выше методики получены значения диэлектрической проницаемости для индивидуальных молекул Нb, которые соответствуют известным литературным данным для белков. Предложенная методика может применяться для изучения диэлектрических характеристик других белковых молекул.

\section{Финансирование работы}

Работа выполнена в рамках государственного задания Омского научного центра СО РАН в соответствии с Программой ФНИ ГАН на 2013-2020 гг. (номер госрегистрации проекта в системе ЕГИСУ НИОКТР ААААA17-117041210227-8).

\section{Конфликт интересов}

Авторы заявляют, что у них нет конфликта интересов.

\section{Список литературы}

[1] Bibi F., Villain M., Guillaume C., Sorli B., Gontard N. // Sensors. 2016. V. 16. N 8. P. 1232. DOI: 10.3390/s16081232

[2] Zhao Yu.D., Bi Y.H., Zhang W.D., Luo Q.M. // Talanta. 2005. V. 65. N 2. P. 489-494. DOI: 10.1016/j.talanta.2004.06.028

[3] Esteban-Ferrer D., Edwards M.A., Fumagalli L., Jurez A., Gomila G. // ACS Nano. 2014. V. 8. N 10. P. 9843-9849. DOI: $10.1021 / \mathrm{nn} 5041476$

[4] Davletkildeev N.A., Stetsko D.V., Bolotov V.V., Stenkin Y.A., Korusenko P.M., Nesov S.N. // Mater. Lett. 2015. V. 161. P. 534-537. DOI: 10.1016/j.matlet.2015.09.045

[5] Staii C., Johnson A.T., Jr. // Nano Lett. 2004. V. 4. N 5. P. 859 862. DOI: $10.1021 / \mathrm{n} 1049748 \mathrm{w}$

[6] Давлеткильдеев Н.А., Соколов Д.В., Лобов И.А., Болотов В.В. // Письма в ЖТФ. 2017. Т. 43. В. 4. С. 47-55. DOI: 10.21883/PJTF.2017.04.44297.16489

[7] Fumagalli L., Gramse G., Esteban-Ferrer D., Edwards M.A., Gomila G. // Appl. Phys. Lett. 2010. V. 96. N 18. P. 183107. DOI: $10.1063 / 1.3427362$

[8] Pethig R., Kell D.B. // Phys. Med. Biol. 1987. V. 32. N 8. P. 933-970.

[9] Simonson T. // Rep. Prog. Phys. 2003. V. 66. N 5. P. 737-787. DOI: $10.1088 / 0034-4885 / 66 / 5 / 202$ 